\title{
Le candidat imaginaire, ou l'offre et le choix dans les élections de la Révolution française
}

\section{Malcolm Crook}

\section{Q OpenEdition}

1 Journals

Édition électronique

URL : https://journals.openedition.org/ahrf/184

DOI : $10.4000 /$ ahrf. 184

ISSN : 1952-403X

Éditeur :

Armand Colin, Société des études robespierristes

\section{Édition imprimée}

Date de publication : 1 septembre 2000

Pagination : 91-110

ISBN : 175 pages

ISSN : 0003-4436

Référence électronique

Malcolm Crook, "Le candidat imaginaire, ou l'offre et le choix dans les élections de la Révolution française », Annales historiques de la Révolution française [En ligne], 321 | juillet-septembre 2000, mis en ligne le 27 avril 2006, consulté le 22 avril 2022. URL : http://journals.openedition.org/ahrf/184 ; DOI : https://doi.org/10.4000/ahrf.184

Ce document a été généré automatiquement le 22 avril 2022.

Tous droits réservés 


\title{
Le candidat imaginaire, ou l'offre et le choix dans les élections de la Révolution française
}

\author{
Malcolm Crook
}

\section{NOTE DE L'ÉDITEUR}

Une première version inédite de cet article a été communiquée à un colloque consacré au suffrage, qui a eu lieu en 1995, sous l'égide du Laboratorio di storia costituzionale de l'université de Macerata.

1 On parle trop facilement de "candidats" dans une histoire électorale de la Révolution française qui est enfin sortie de l'ombre 1 . Des candidats dans le sens moderne du terme n'existent pas ou, plus exactement, à la différence de nos jours on ne retrouve pas au cours de la décennie révolutionnaire de candidats déclarés, sauf en l'an $\mathrm{V}$ de la République. Car, malgré la pratique des candidatures "officieuses", qui préfigure l'idée de la compétition électorale et annonce un nouveau concept du candidat, il persiste dans l'imaginaire politique des Français de cette époque un personnage idéal à élire, un homme qui convient parfaitement au poste, mais qui refuse de se mettre en avant. Ce candidat n'a pas besoin de publicité ni de programme, au contraire, solliciter des suffrages lui attirerait du discrédit. Ce refus obstiné de la pratique des candidatures déclarées nous révèle donc clairement la façon de penser l'offre et le choix dans les élections de la Révolution.

2 Pour reprendre la formule d'Aulard "les mœurs répugnaient encore aux candidatures, de même qu'on avait honte de se dire d'un parti"'. Selon l'instruction du 28 mai 1790, on doit afficher dans la salle d'assemblée électorale l'avis suivant:

"Vous jurez et permettez de ne nommer que ceux que vous aurez choisis en votre âme et conscience, comme les plus dignes de la confiance publique, sans avoir été déterminé par dons, promesses, sollicitations ou menaces." ${ }^{3}$ 
“il sera constaté qu'il y a eu supposition de suffrages, ou qu'ils ont été captés par des voies illicites"4. L'absence de candidats déclarés sous la Révolution représente en effet un héritage de l'Ancien Régime, encore un élément archaïque aux origines sans doute ecclésiastiques, qui soulève nombre de problèmes dans la gestion du nouveau système électoral.

4 En effet, la dispersion des voix et le refus des élus de servir donnent matière à réflexion et la Constitution de l'an III rend possible la candidature déclarée. Expérience unique, mise en pratique une seule fois en 1797, cet épisode mal documenté et à peu près inconnu mérite un examen approfondi. La déclaration des candidatures est une innovation du Directoire, mais son abolition abrupte l'année suivante est également révélatrice d'une mentalité adverse qui se méfie toujours de la concurrence aux élections. Cette idée est tellement bien enracinée dans l'imaginaire français qu'on devra attendre encore un siècle pour que la déclaration des candidatures devienne obligatoire. Tradition de longue durée, donc, que l'événement révolutionnaire, dans ce domaine comme dans beaucoup d'autres, ébranle sans l'abolir définitivement.

I

5 En ce qui concerne le système électoral, 1789 ne constitue pas du tout une année zéro. Les Constituants empruntent beaucoup à l'Ancien Régime qui, au niveau local, maintient tout au long du xvIIIe siècle une tradition dont la richesse et la diversité sont trop souvent ignorées par les historiens ${ }^{5}$. Cet héritage sert de base pour les élections aux États généraux de $1789^{6}$. Certes, l'année suivante, les députés de la Constituante abolissent le vote par ordre et par corporation - rupture profonde dans l'histoire des élections - mais sous la Révolution le citoyen doit toujours voter en assemblée, mécanisme traditionnel où dominent l'esprit et la pratique communautaires.

Le refus des candidats déclarés fait partie de cette continuité électorale, entravant ainsi l'émergence des mentalités nouvelles. L'élu doit représenter la collectivité, non pas une faction particulière. L'instruction qui accompagne la célèbre réforme municipale de Laverdy, en 1765, insiste donc sur la nécessité de ne pas empêcher "le choix le plus éclairé" . L'exemple des candidats aux élections britanniques de la même époque (déterminées par "les promesses et les pots de bière), confirme les Français dans leur option plutôt traditionnelle ${ }^{8}$. On peut observer, cependant, que la pratique anglaise du début du xviIe siècle est parfaitement conforme au modèle de l'Ancien Régime ; c'est à la suite de la révolution des années 1640 que le processus d'élection y devient plus compétitif 9 .

7 Les assemblées électorales françaises du xviIIe siècle sont composées des notables urbains dans les villes, et des chefs de famille à la campagne, milieux restreints, même intimes, où tout le monde se reconnaît. On laisse souvent au titulaire sortant le droit de nommer son successeur il présente son choix à l'approbation générale, tandis que le hasard joue aussi un rôle dans la désignation. La Révolution par contre établit des assemblées cantonales ou départementales, ainsi que municipales, réunions plus grandes et plus anonymes, où le processus électoral prend une tout autre allure. On peut estimer le nombre de postes à pourvoir en 1790 à près d'un million, tandis que plus de 4000000 de citoyens ont le droit de voter. L'éligibilité est assez étendue à tous les stades (seul l'accès à la députation nationale reste très restreint en 1791) et la conséquence, pour emprunter l'expression de Patrice Gueniffey, est de plonger les votants, confrontés à un choix illimité, "dans le vide"

Annales historiques de la Révolution française, 321 | juillet-septembre 2000 
8 Les campagnards au collège électoral de l'Eure-et-Loir en 1790 se plaignent avec raison: comment choisir les membres du Conseil général du département quand on ne connaît presque personne parmi les autres électeurs ${ }^{11}$ ? L'absence des candidats déclarés apporte avant tout une extrême dispersion de voix. À Toulon, dans les élections municipales inaugurales de 1790 , une trentaine d'individus sont proposés pour le poste de maire et pas moins de 461 noms sont enregistrés lors du premier tour de scrutin pour les vingt-huit notables (ou conseillers adjoints) ; presque chaque votant se nomme lui-même sur son propre bulletin ${ }^{12}$ ! Même comportement au Puy, où 290 votants nomment 568 individus et à Strasbourg, où le total des nominations s'élève à $1737^{13}$. Très peu d'élus au premier tour, ainsi qu'au deuxième, généralement inutile, car on annonce tout simplement que "personne n'a obtenu la pluralité absolue", et la concentration des voix s'avère difficile. Quand la pluralité relative suffit au troisième et dernier tour du scrutin de liste, il n'est pas rare qu'on soit nommé par une poignée de voix.

9 Et pourtant, l'objet visé consiste à obtenir l'approbation de la majorité des votants pour tous les titulaires. Dans le cas des scrutins uninominaux, il est vrai que, faute de majorité absolue à la suite du deuxième tour, on prend les deux individus les mieux placés pour un troisième et dernier scrutin de ballottage. Plus de la moitié des évêques élus en 1791, par exemple, le sont au troisième tour. Le choix se limite enfin, mais il doit tomber sur l'un ou l'autre de ces "candidats" (on se sert du mot dans ce sens précis). À cause des caprices du choix illimité des deux premiers tours de scrutin, ce processus peut déboucher sur l'élection d'une personne ayant attiré peu de voix au début du concours. Gueniffey cite l'exemple des élus à l'Assemblée législative de 1791 dans les Hautes-Alpes, qui ont recueilli moins de $10 \%$ des voix lors du premier tour de scrutin. Plutôt que d'exprimer leur préférence, les électeurs se résignent à un pisaller ${ }^{14}$. Devant un tel choix, on comprend pourquoi beaucoup de participants décident de s'abstenir.

10 Le problème de la dispersion des voix est exacerbé en 1790 par la pratique, vite abandonnée, de la double liste. Sauvegarde théorique contre la cabale, cette procédure oblige les votants à écrire deux fois le nombre d'individus à élire. Ceci pose de graves difficultés pour les participants, qui sont obligés de proposer beaucoup de noms, et d'autant plus pour les scrutateurs, qui doivent déchiffrer une masse de noms souvent illisibles et impossibles à distinguer. Il faut souligner que l'utilisation de bulletins imprimés, ou écrits d'avance, est strictement interdite jusqu'en l'an V : “Tout bulletin qui aura été apporté dans les assemblées et qui n'aura pas été, ou écrit par le votant luimême sur le bureau, ou dicté par lui aux scrutateurs s'il ne sait pas écrire, sera rejeté comme nul." 15

11 À Parizot, dans l'Aveyron, les scrutateurs s'excusent de la perte d'une journée occupée uniquement au dépouillement du scrutin. Ce délai est occasionné par "la dénomination diversifiée; presque sur chaque bulletin des sujets différents" ${ }^{16}$. La situation est pire à Paris où, dans une section comme les Quatre-Nations, avec ses 3900 citoyens actifs dont 500 votent, lesscrutateurs ont affaire en 1790 à quelque 20000 nominations ${ }^{17}$. Toujours dans la capitale, mais l'année suivante au sein de l'assemblée départementale de Paris, une grande dispute s'élève au sujet de l'identité d'un certain Monneron, désigné de plusieurs façons ${ }^{18}$. En 1792, dans l'Oise, confusion totale autour de deux Bourdon, émanant tous les deux de Paris et qu'il est impossible de distinguer l'un de l'autre sur les bulletins ${ }^{19}$. 
12 l'appellent, est encore un inconvénient découlant de l'absence de candidatures déclarées. Le retrait impose souvent un deuxième scrutin pour trouver un remplaçant : l'ancien intendant du Languedoc est choisi comme maire de Montpellier en 1790 mais, sur son refus, on est obligé de convoquer de nouveau les citoyens le mois suivant. Le problème est pire aux élections épiscopales de 1791 : dans l'Orne, l'élection d'un évêque nécessite cinq scrutins ${ }^{20}$. De tels cas se produisent dans plusieurs départements à cause des non-acceptations : dans les Deux-Sèvres, à la suite d'un deuxième refus, le Club des jacobins de Niort demande que le démissionnaire rembourse aux électeurs leurs frais de déplacement ${ }^{21}$. Pareil cas pour les députés nationaux : "Hier [raconte un électeur de la Seine-Inférieure en 1792] nous avons perdu tout notre temps. Nous avions nommé deux députés [...] qui ont donné l'un et l'autre leur démission." ${ }^{22}$ citoyen de Ploëmerl, dans le Morbihan, est convaincu que son élection résulte de la mauvaise volonté de ses voisins. Il n'a pas participé à l'assemblée cantonale et, à l'âge de soixante-dix-huit ans, il n'a pas d'envie d'assister à l'assemblée départementale non plus. Sa demande que "l'on lelaisse mourir tranquillement chez lui" est néanmoins rejetée ${ }^{23}$. Gueniffey évoque le cas d'un certain Fabre, qui ignore son choix comme électeur de deuxième degré à l'assemblée départementale de l'Aude en 1791. Après avoir rencontré un ami dans les rues de Carcassonne, qui l'avertit de son élection, il arrive en retard à l'assemblée et présente ses excuses ${ }^{24}$ ! Dans ces circonstances, une pression est souvent exercée contre des élus peu disposés à servir. Pour éviter une nouvelle réunion de leur assemblée municipale, les habitants du village d'Aigre, dans la Charente, vont chez le maire élu "et tous d'unanime voix lui ont demandé avec insistance d'accepter, ce qu'il a fait pour obliger ladite commune." ${ }^{25}$

Un moyen de prévenir les démissions ou le choix de personnes inaptes est de nommer des hommes expérimentés déjà en place. Les électeurs de deuxième degré, qui siègent aux collèges électoraux dans les départements, sont souvent choisis parmi les maires, conseillers municipaux ou juges de paix. Dénoncés comme une "aristocratie invisible" qui monopolise les postes, ces hommes constituent des représentants naturels, parce qu'ils sont des personnages en vue, les seuls capables, ou à faire preuve de bonne volonté au moins. Ils représentent la communauté au niveau le plus élevé du processus électoral. Jean-Pierre Jessenne fait remarquer à juste titre que "..Beaucoup de maires sont aussi électeurs, comme si cette fonction était perçue comme le prolongement logique du mandat local et des tâches de représentation de la collectivité qu'il implique" ${ }^{26}$. Quand il s'agit de choisir les administrateurs départementaux en 1790, la plupart des élus se recrutent parmi ces électeurs, à tel point qu'un citoyen anonyme de la section des Enfants-Trouvés à Paris, proteste : "Beaucoup de citoyens s'efforcent de devenir électeurs, non dans l'ambition de bien choisir, mais dans l'espoir d'être choisis eux-mêmes." ${ }^{27}$

L'absence de candidats déclarés nécessite toujours un concours électoral (et pendant la Révolution, il y en a beaucoup, même trop, sans grande participation si le résultat est connu d'avance). En Angleterre au xviIIesiècle, par contre, des sièges parlementaires ne sont pas disputés, en raison d'une candidature unique ${ }^{28}$. Malgré leur méfiance vis-à-vis de la pratique anglaise, certains Français reconnaissent le problème ainsi posé par un choix électoral illimité2 ${ }^{9}$. Condorcet propose un scrutin de présentation, afin d'établir une liste de candidats au scrutin définitif. Brissot demande la publicité, soit dans la 
presse (qui se limite en 1791 à recommander "de bons patriotes"), soit par la lecture des résultats du premier scrutin, moyen d'indiquer les "leaders" et ainsi d'encourager la concentration des voix. Il est aussi partisan de la création de "clubs électoraux" où les électeurs pourraient ouvertement discuter les mérites des citoyens aptes à servir. Il adhère à une société d'électeurs patriotes, établie quand l'assemblée électorale de Paris se réunit en novembre 1790, afin de "s'éclairer mutuellement sur les choix importants que les électeurs ont à faire", mais en ajoutant "sans que l'opinion la plus générale puisse gêner l'opinion particulière de ses membres dans l'assemblée électorale" ${ }^{30}$. Tous les électeurs n'y sont pas admis et l'année suivante, à la suite d'un différend, il y a deux clubs, un séant à l'Évêché, l'autre à la Sainte-Chapelle; la volonté générale se trouve divisée. Un des participants, un certain Nau-Deville, s'en inquiète profondément: "N'est-il pas vrai que si quelqu'un disait tout haut, je nomme... tel, en ma conscience, il pourrait influencer, entraîner le vœu de celui qui l'entendrait ?"31

Brissot affirme la nécessité de la publicité et de la discussion car en réalité des "candidats", plus ou moins cachés, entrent en lice. Bien sûr, sous l'Ancien Régime, brigues et cabales se produisaient; la preuve, c'est qu'elles furent défendues. Même chose en 1789 où, à l'approche des élections aux États généraux, apparaît un Avis au Tiers État de Paris, justifié par "le devoir d'un bon patriote d'indiquer les hommes instruits et connus par leur amour du bien général" ${ }^{32}$. Normalement ce sont des parents ou des amis qui font campagne électorale, car solliciter soi-même des voix aurait pour effet de se discréditer (et clamer son incapacité serait presque un moyen de se recommander!) $)^{33}$. Selon un citoyen de la Drôme, "toute la propagande se faisait par des amis en conciliabules privés", moyens qui par leur nature même laissent peu de traces pour l'historien ${ }^{34}$.

17 Visites et invitations se succèdent, à la recherche de promesses avant l'ouverture de l'assemblée et, selon un pamphlet satirique, les électeurs ruraux sont les plus faciles à séduire de cette manière, aux approches du chef-lieu ou dans les auberges. Les assemblées électorales durent plusieurs jours et offrent maintes occasions de faire campagne parmi les participants qui s'y présentent. Selon l'auteur anonyme de la même brochure, on doit assister à toutes les petites discussions de groupe qui ont lieu pendant l'appel au scrutin ou durant le dépouillement des voix. Participer à la direction des affaires représente encore un moyen de se faire élire: "À l'ouverture de l'assemblée, on se trouve comme par hasard au bureau ; on y taille négligemment ; c'en est assez pour être fait secrétaire"; mais il ne faut pas démontrer "trop d'ambition" ${ }^{35}$.

La concentration des voix au premier tour, tout comme les plaintes des non-élus, démontrent bien l'existence de ces pratiques électorales officieuses. À Toulon, où l'indiscipline des votants est bien évidente en 1790, une transformation s'annonce l'année suivante : au lieu de trente nominations pour le poste de maire, il n'en reste que quatre et presque toutes les voix (98\%) sont données à deux "candidats" provenant des clubs rivaux ${ }^{36}$. En effet, à partir de l'été 1791, les sociétés politiques pratiquent la confection des listes, l'envoi de commissaires ou agents électoraux et la convocation de réunions pour la discussion des "candidats". Le club de Beauvais, par exemple, publie une liste des citoyens à élire à l'assemblée départementale de l'Oise, tandis que dans ceux de Lille et de Toulouse circulent les noms de ceux qu'il faut éviter à tout prix ${ }^{37}$.

Dès le début de la Révolution on s'efforce de répartir les postes à pourvoir entre les différentes communes d'un canton, ou parmi les districts d'un département, afin d'assurer une représentation de tous les participants. Cette stratégie facilite le choix 
aux conseils départementaux en 1790 : dans l'Eure-et-Loir, par exemple, on réussit à élire tous ces administrateurs au premier tour de scrutin. À partir de 1791, par contre, il ne s'agit plus de trouver le "meilleur représentant de la communauté, mais plutôt le porte-parole des opinions diverses ou des partis opposés, non sans crainte de voir surgir faction et division. À Marseille, au début de 1793, une protestation révèle clairement la mise en œuvre d'une véritable campagne électorale et, en même temps, l'horreur d'une telle pratique :

"Nombre de sections de cette ville ont nommé des commissaires qui se sont réunis en diverses séances au ci-devant couvent des Dominicains, et que là ils y ont formé une liste de vingt officiers municipaux, qu'ils l'ont ensuite porté cette même liste, chacun d'eux dans leur section respective, et que certains, et c'est le grand nombre, ont été forcés de suivre cette même liste ou par erreur ou par crainte, et que cette voie est entièrement illégale $[. . .]^{38}$

Malgré ses réticences initiales la presse parisienne, sinon celle de la province, commence aussi à donner des conseils précis aux votants. L'Ami du peuple de Marat et $L a$ Sentinelle de Louvet, parus tous les deux sous forme d'affiches en août 1792, n'hésitent plus à faire un compte rendu critique des candidats à la Convention nationale ${ }^{39}$. Il existe, donc, un autre discours sur l'offre et le choix électoral, qui approuve la recherche de voix en faveur des candidats qui représentent des options politiques opposées. Devant ces constatations, on ne saurait se ranger au verdict de Gueniffey qui dénonce "les stratégies minoritaires" comme la négation du vote ${ }^{40}$. Ce sont plutôt des preuves d'une évolution vers le concept, ou plutôt la pratique de la compétition électorale, voire le pluralisme politique.

21 La méfiance contemporaine vis-à-vis de ces innovations dans le comportement électoral persiste quand même. De chaque côté, l'on continue à s'opposer aux candidatures ouvertes : un militaire qui apporte son aide à l'élection de Dubois-Crancé à la Convention nationale est vivement critiqué comme "cabaleur" dans le journal jacobin de Marseille ${ }^{41}$. À l'assemblée départementale de l'Oise, toujours en 1792, on propose la discussion d'une liste de candidats, mais sans succès "par crainte de porter atteinte à la liberté des suffrages" ${ }^{42}$. La statistique des voix témoigne également de la résistance des électeurs de deuxième degré aux pressions partisanes : il est bien vrai qu'en 1792 presque la moitié des députés à la Convention nationale sont élus au premier tour de scrutin (contre le tiers en 1791), mais en revanche deux députés sur cinq ne sont élus qu'au troisième et dernier tour ${ }^{43}$.

II

Dans les projets soumis pour guider les rédacteurs de la célèbre Constitution de 1793, la notion de la candidature déclarée devient cependant plus répandue. Plusieurs propositions avancent l'idée d'un scrutin "préparatoire" pour établir une liste de candidats qui serait présentée lors du scrutin "définitif". Duplantier, député de la Gironde, va plus loin en proposant qu'on distribue aux citoyens :

"la liste imprimée des candidats, qui aurait été déjà affichée pendant un mois dans chaque commune, afin de donner le temps aux citoyens de les connaître et d'apprécier leurs talents et leurs vertus. Sur cette liste chacun, après y avoir marqué en même temps et à un signal convenu ceux qu'il préfère, la porterait [...] dans l'urne $[. . .]^{\prime \prime 4}$

Il est intéressant de noter que ce projet persiste à concevoir le vote comme un geste collectif. Il reste bien rare de trouver l'idée d'une participation individuelle, même isolée, à l'abri de l'intimidation ; quand J.-V. Delacroix, rédacteur du Spectateur français, 
suggère en 1795 qu'on remplisse son bulletin de vote dans "une chambre particulière", on le dénonce à la Convention ${ }^{45}$. Mais Duplantier n'est pas le premier à penser à des listes de candidats: Choderlos de Laclos, aux Jacobins de Paris, avait déjà proposé l'ouverture de registres, "où les aspirants pourraient se faire inscrire" ${ }^{46}$. Au même instant les jacobins de Quimper demandaient un pareil système d'inscription publique, afin d'éviter les tours successifs de scrutin, les voix perdues et aussi pour détourner les gens "inciviques" 4 .

Ce n'est pas avec la Constitution démocratique de 1793, jamais appliquée, mais plutôt avec celle plus conservatrice de 1795 qu'on adopte ce type de candidatures. Selon la loi électorale du 25 fructidor an III (11 septembre 1795),

"durant le mois de nivôse [décembre - janvier], chaque citoyen a le droit de se faire inscrire lui-même, ou de faire inscrire ceux de ses concitoyens qu'il juge à propos, sur la liste des candidats [...] pour une ou plusieurs des fonctions qui sont à remplir dans le mois de germinal [mars - avril] suivant [l'époque annuelle des élections sous le Directoire]".

Ces candidatures sont reçues par l'administration municipale, qui se contente de vérifier les qualités d'éligibilité, avant de passer à l'administration supérieure les nominations pour les postes à pourvoir par l'assemblée départementale. Les listes à présenter aux assemblées, primaires ou secondaires, sont publiées durant les mois de pluviôse et de ventôse qui précèdent les élections, affichées et lues dans les salles de réunion en germinal. À l'institution des candidatures déclarées il manque, cependant, la rigueur du projet Duplantier, car les votants restent toujours libres de porter leur voix sur n'importe quel citoyen non compris dans la liste.

"Cette manière franche de s'offrir à la confiance est la plus digne d'un républicain, et, sous tous les rapports, elle est préférable aux brigues secrètes et aux manœuvres obscures de l'ambition intrigante", prononce une instruction complémentaire du 5 ventôse an V (23 février 1797). Selon les administrateurs du Lot-et-Garonne, c'est "pour tous les citoyens un moyen de désigner à l'opinion publique les sujets qu'ils ne peuvent pas élire directement", fonction réservée aux électeurs de deuxième degré sous le système électoral indirect de la décennie révolutionnaire ${ }^{48}$. En même temps que les candidatures, on introduit au second et dernier tour d'élection un système de double vote, l'un de nomination, l'autre de rejet, pour empêcher "qu'un candidat ne soit jamais élu contre le gré de la majorité des électeurs" ${ }^{49}$. Après le premier tour on ne retient qu'un nombre de nominations décuple de celui des postes restant à pourvoir, limitant ainsi l'offre au deuxième tour, car les noms publiés deviennent les seuls en concurrence.

L'introduction du nouveau système électoral de l'an III sera reportée à l'an V, faute d'exécution immédiate (en l'an III les conventionnels sortants, dont les deux tiers doivent être réélus, sont de fait des candidats). En effet, l'expérience des candidatures déclarées de l'an $\mathrm{V}$, qui fait partie de toute une série d'innovations remarquables introduites sous le Directoire, reste unique et, jusqu'à la parution de l'excellent livre de Patrice Gueniffey sur les élections de la Révolution française, elle était passée presque inaperçue par les historiens (comme l'histoire du Directoire en général). Pour approfondir et nuancer le commentaire suggestif qu'on trouve dans Le nombre et la raison de Gueniffey, il serait nécessaire d'élargir les recherches. Malheureusement les candidatures de l'an $\mathrm{V}$ ne laissent pas souvent de traces aux archives et l'analyse qui suit se borne, pour l'essentiel, à sept listes départementales en provenance de la Côte- 
d'Or, de la Gironde, de la Loire-Inférieure (Atlantique), du Lot-et-Garonne, de la Meurthe, de la Seine-et-Oise (Yvelines) et de la Haute-Vienne ${ }^{50}$.

Force est de constater qu'au stade primaire des élections, où s'élisent électeurs de deuxième degré, juges de paix et personnel municipal, la réponse est bien mince: quatre-vingt-une nominations pour tous ces postes à Salers, dans le Cantal et soixantesix à Sèvres, dans la Seine-et-Oise; mais pas plus que quarante-six à Nantes et seulement une trentaine à Strasbourg ${ }^{51}$. Hors des villes, semble-t-il, moins de candidatures sont enregistrées : six propositions dans deux autres cantons du Cantal et une douzaine dans deux cantons des Côtes-du-Nord (Côtes-d'Armor) ${ }^{52}$.

Gueniffey observe que dans les cantons ruraux de la Seine-et-Oise le registre reste "parfois vierge de toute inscription", mais on y trouve souvent mention de la lecture des listes dans les procès-verbaux ${ }^{53}$. Dans le Finistère, par contre, la mention de candidats est très rare et se remarque surtout par son absence : à Guerlesquin, par exemple, on écrit "nous n'avons pas de candidats pour les fonctions publiques; personne ne s'est présenté pour en nommer"54. À Riez, dans les Basses-Alpes (Alpes de Haute-Provence), le président de l'assemblée demande un exemplaire de la liste, mais on lui répond qu'il n'en existe pas, faute de candidats ${ }^{55}$. Dans le Lot-et-Garonne l'administration départementale critique les municipalités parce qu'elles ont négligé les préparatifs d'inscription ${ }^{56}$. Dans la Loire-Inférieure, infestée de chouans, il y a également des cantons ruraux où l'on n'a pas réussi à ouvrir de registre ${ }^{57}$.

Dans ce dernier département, seuls onze cantons sur cinquante-cinq hors de Nantes avaient déposé des nominations au stade secondaire (législateurs, administrateurs départementaux, personnel judiciaire). Néanmoins, on recense ici un total de 259 nominations, dépassant d'une courte tête la Côte-d'Or (249) et nettement plus que la Gironde (92), ou la Haute-Vienne (34), mais moins que les 279 du Lot-et-Garonne, les 484 de la Meurthe, les 637 de la Seine-et-Oise et, bien sûr, les 1100 indiquées à Paris (où l'on renonce à lire la liste de tous les noms à cause de sa longueur) ${ }^{58}$. Voici la répartition de ces candidats dans les sept départements dont les listes se trouvent aux archives :

\begin{tabular}{|c|c|c|c|c|c|c|c|c|}
\hline \multirow[t]{2}{*}{ Département } & \multicolumn{2}{|c|}{ Corps admin. } & & & & \multirow{2}{*}{\multicolumn{2}{|c|}{ Tribunaux }} & \multirow{3}{*}{ Total } \\
\hline & \multicolumn{2}{|c|}{ législatif dépt. } & & & & & & \\
\hline & & no & (\%) & no. & (\%) & no. & (\%) & \\
\hline Côte-d'Or & & 82 & $(30,8)$ & 142 & $(53,4)$ & 42 & $(15,8)$ & 266 \\
\hline Gironde & & 45 & $(48,9)$ & 37 & $(40,2)$ & 10 & $(10,9)$ & 92 \\
\hline Loire-Infériet & ure & 113 & $(43,6)$ & 52 & $(20,1)$ & 94 & $(36,3)$ & 259 \\
\hline Lot-et-Garon & & 87 & $(31,2)$ & 102 & $(36,6)$ & 90 & $(32,2)$ & 279 \\
\hline Meurthe & & 157 & $(32,4)$ & 165 & $(34,1)$ & 162 & $(33,5)$ & 484 \\
\hline
\end{tabular}




\begin{tabular}{|l|l|l|l|l|l|l|l|}
\hline Seine-et-Oise & 240 & $(35,9)$ & 279 & $(41,8)$ & 149 & $(22,3)$ & 668 \\
\hline \hline Haute-Vienne & 15 & $(44,1)$ & 15 & $(44,1)$ & 4 & $(11,8)$ & 34 \\
\hline \hline Total & 739 & $(35,5)$ & 792 & $(38,0)$ & 551 & $(26,5)$ & 2082 \\
\hline \hline $\begin{array}{l}\text { NB Les mêmes personnes sont souvent proposées à plusieurs postes : dans la Meurthe, par } \\
\text { exemple, les 484 nominations concernent 226 individus. }\end{array}$
\end{tabular}

L'intérêt porté aux candidatures est donc très variable d'un département à l'autre, mais on n'a pas besoin d'en chercher une explication profonde. L'exemple de Nantes, étudié par Yvon Le Gall dans sa thèse qui a fait date sur les consultations électorales dans la Loire-Inférieure sous la Révolution, semblerait indiquer que les inscriptions auraient été faites par une poignée d'amis ou de collègues, parce qu'un individu a le droit d'enregistrer plusieurs noms à la fois. Ainsi un administrateur propose des confrères, un négociant fait de même et, chose plus curieuse, "une mini-campagne est organisée en faveur de quatre colons de Saint-Domingue, vraisemblablement par d'autres colons" 59 .

Ce qui ressort clairement des listes, c'est la domination des chefs-lieux de département : presque la moitié des propositions de la Loire-Inférieure vient de Nantes et en Seine-et-Oise la même proportion provient de Versailles; dans la Gironde, plus du tiers des candidats résident à Bordeaux, tandis qu'en Côte-d'Or le quart en est domicilié à Dijon. La composition socio-professionnelle des inscrits est également intéressante, dans les cas où elle est notée. La prépondérance des professions libérales par rapport au commerce est particulièrement frappante, même dans les deux départements maritimes, la Gironde et la Loire-Inférieure. Pas un seul négociant inscrit pour le corps législatif dans celle-là, pas plus que sept dans celle-ci. Dans les autres départements les hommes de commerce font également défaut comme législateurs. On retrouve une réticence semblable de la part des négociants vis-à-vis de l'administration départementale et, bien sûr, envers les tribunaux.

Hommes de loi, notaires et propriétaires sont majoritaires et il est naturellement bien rare de trouver mention d'un agriculteur ou d'un artisan, ceux-ci étant proposés uniquement pour l'administration départementale. Par contre il faut souligner la forte présence d'hommes qui remplissent déjà des postes électifs comme officiers municipaux ou juges de paix, par exemple: dans la Meurthe plus de la moitié des inscrits sont dans ce cas. Les fonctionnaires s'y manifestent, surtout les commissaires du pouvoir exécutif le commissaire près l'administration centrale de la Loire-Inférieure est en effet désigné par six cantons. C'est le reflet du rôle de plus en plus important de ces fonctionnaires dans la République. Ce sont des hommes en vue, comme les députés ou administrateurs de la première heure toujours en poste et les juges, que le régime directorial s'empresse d'utiliser dans les élections.

Il faut aussi remarquer l'inscription des "grandes vedettes" de réputation nationale, car on peut choisir des députés hors du département : le directeur Barras dans le Lot-etGaronne et le ministre Talleyrand en Côte-d'Or; Bonaparte et plusieurs autres militaires (y compris Pichegru, nommé en même temps dans le Cantal, la LoireInférieure, le Bas-Rhin et la Seine-et-Oise) ; Bougainville, "célèbre voyageur", Bernardin 
de Saint-Pierre, “auteur des études de la nature", ainsi que La Harpe et Bonneville, "hommes de lettres", dans la Loire-Inférieure ; et en Haute-Vienne on retrouve le nom de Pierre-Antoine Antonelle, ex-marquis traduit "en jugement devant la Haute Cour de Justice séante à Vendôme" pour sa participation à la conspiration de Babeuf !

Il y a beaucoup, voire trop de candidats, pour un nombre restreint de places quelques députés à élire aux Conseils, cinq administrateurs au maximum aux directoires départementaux; en moyenne, donc, on retrouve une trentaine de nominations par place. Les 300 ou 400 électeurs dans chaque département étudié, se trouvent devant un embarras de richesses, ou plutôt un choix impossible. En fin de compte ils peuvent voter pour n'importe qui, outre les candidats inscrits, comme aux élections précédentes. L'autorisation du candidat proposé n'est pas nécessaire non plus, ce qui explique le refus de Morizot de représenter la Côte-d'Or aux Cinq-Cents et la présence de Baco sur la liste nantaise, malgré sa récente démission comme député. En Côte-d'Or, il est vrai, la plupart des élus sont pris sur la liste de candidats, mais c'est le contraire en Loire-Inférieure où aucun des députés élus n'est inscrit candidat.

Le Gall est persuadé que les royalistes de la Loire-Inférieure n'utilisent pas la candidature déclarée pour faire leur campagne. Une liste saisie sur un ancien chef de chouans contient vingt-quatre noms pour la législature, mais on ne retrouve que quatre de ces noms sur celle qui est publiée ${ }^{60}$. Reinhard fait la même constatation pour la Sarthe où les listes officielles affichent des noms républicains ${ }^{61}$. En Loire-Inférieure, pourtant, l'attachement à la République de la part des notables enregistrés comme candidats suffit pour les faire échouer: le commissaire central, par exemple, est éliminé lors du scrutin d'exclusion, malgré plusieurs nominations, car il est le meneur de la minorité patriote dans une assemblée qui manifeste une nette hostilité envers le régime républicain.

III

L'expérience des candidatures déclarées laisse donc beaucoup à désirer. Elle ne résout pas les problèmes de l'offre et du choix électoraux, car les voix sont toujours dispersées et il y a des élus qui refusent de servir. Avant tout, les votants sont désemparés, confrontés à trop de noms et pouvant soutenir n'importe qui. En plus, le système ne fonctionne qu'au niveau des assemblées secondaires du département ; il est ignoré dans l'écrasante majorité des cantons. Néanmoins on fait un pas vers l'innovation. Le système est susceptible de modification aux élections suivantes et l'on peut s'attendre à une évolution dans la sensibilité des participants. Mais cela reste sans effet, car l'année suivante, lors des élections de l'an VI, les candidatures déclarées sont supprimées et l'expérience ne sera pas répétée.

Selon Pons de Verdun, dans son rapport sur les listes de candidats, communiqué au Conseil des Cinq-Cents le 21 nivôse an VI (10 janvier 1798), leur suppression est "vivement désirée par tous les républicains", parce qu'elles "renferment un germe de division et de scandale" ${ }^{2}$. "Ni pleinement facultatives, ni pleinement impératives", les listes sont contradictoires et favorisent l'intrigue, car "l'amour propre d'un galant homme sera toujours choqué par l'idée d'aller quêter les regards de ses concitoyens". Avant tout, il cite "la préférence qu'elles ont donnée jusqu'ici à des conspirateurs, au préjudice des républicains". Sur ce dernier point, Pons a tort. Comme l'on vient de le voir, les ennemis de la République ne se servent guère des listes. La défaite du Directoire lors des élections de l'an V reflète tout simplement une répudiation des anciens conventionnels sortants des Conseils, voire une hostilité implacable vis-à-vis 
du régime établi en 1795. Les candidats servent de boucs émissaires pour la défaite électorale sans doute inévitable de 1797.

Lebreton, dans un rapport au Conseil des Anciens délivré le 24 pluviôse (12 février 1798), est aussi convaincu que Pons des conséquences désastreuses des listes ${ }^{63}$. Il considère l'expérience historique des candidatures avant de conclure que "le candidalat a perdu la République romaine et il perdrait la République française". Les bons citoyens sont exposés "à la méchanceté, aux sarcasmes et à de véritables injures", tandis que les listes "n'ont servi qu'à ces hommes qui ont nécessité le 18 fructidor" (l'épuration des nouveaux élus des Conseils en septembre 1797). Il cite Jean-Jacques Rousseau comme témoin en déclarant que "les électeurs n'ont besoin que de leurs consciences."

Comme celui de Lebreton, le rapport de Pons est surtout révélateur des mentalités profondément enracinées dans l'imaginaire politique français, qui reste très méfiant à l'idée de candidats déclarés. En exhortant les législateurs à "rendre la liberté aux assemblées", Pons ajoute ces mots extraordinaires :

"Le désir de faire connaître et d'élever aux places, par son suffrage, celui qui en est digne n'est, pour ainsi dire, qu'un germe dans l'âme de chaque votant : c'est dans les réunions, dans les grandes assemblées, qu'il se développe et qu'il éclate. Là, par l'effet de je ne sais quelle électricité morale composée de toutes sortes d'éléments, la majorité éprouve une commotion dont elle ne peut se défendre; il lui vient une pensée commune, et elle fait pour la justice ce que chacun de ses membres n'aurait pas fait isolément."

41 Cet utopisme l'emporte sur l'intérêt politique dans la suppression des candidats déclarés, parce qu'il est partagé de part et d'autre.

Un des nouveaux élus aux corps législatifs en l'an $\mathrm{V}$, le conservateur Quatremère de Quincy, avait déjà écrit une diatribe intitulée $L a$ véritable liste de candidats pour s'opposer au système, qui pour lui ne produirait que des disputes et encouragerait des concurrents faibles ${ }^{64}$. Les listes de candidats, continue-t-il, "paraissent moins faites pour calmer ces agitations que pour les accroître", car "la véritable candidature, au contraire, [est] celle de l'opinion publique". Or :

"La véritable liste des candidats... devrait consister, non pas dans un tableau de noms exposés à la critique, mais dans le tableau raisonné des principes sur lesquels devrait se fonder cette critique. La vraie table des candidats devrait être, non la liste où tous les partis s'inscrivent, mais cette série de notions et de règles qui dispenseraient de liste et de candidats. Ce serait celle qui donnerait à tous le signalement des hommes à élire...."

Quatremère propose l'élection de personnes "éprouvées dans les événements de la Révolution, "moralement éligibles" en vertu de leur conduite durant les années précédentes. En même temps, il restitue la notion des "mandats" à donner aux élus, tradition toujours vivace dans l'imaginaire électoral et moyen de rendre "le bon choix" moins impératif. Quoique "fructidorisé" en septembre 1797, Quatremère tombe d'accord avec Pons, son adversaire politique à quoi bon les candidatures déclarées ?

En l'an VI, en même temps que les listes de candidats, on supprime le scrutin de rejet, autre innovation de l'an $\mathrm{V}$, pour rétablir un scrutin majoritaire à trois tours ${ }^{65}$. L'utilisation des bulletins de rejet a semé la confusion chez les électeurs de la HauteVienne, par exemple, et ce scrutin essaie d'empêcher l'élection du "mauvais", plutôt que de faciliter le triomphe du "bon" candidat ${ }^{66}$. La mise aux urnes de bulletins écrits d'avance, ou imprimés, est aussi de nouveau défendue. On revient donc aux pratiques 
désuètes des années précédentes. Selon le Directoire exécutif, dans son adresse au peuple à la veille des élections de 1798 , le bon choix s'exercerait dans une liberté complète et distinguerait facilement le citoyen vertueux, qui se tient toujours à l'écart des partis et ne cherche pas à s'imposer. En réalité, les minorités agissantes, et même l'Exécutif lui-même, essaient d'en déterminer le résultat; l'offre et le choix électoraux ne seront pas tout à fait libres, quoiqu'on prétende le contraire ${ }^{67}$.

C'est l'heure des Cercles constitutionnels, de ces "néo-jacobins qui cherchent à renverser la poussée "royaliste" de l'an $\mathrm{V}$ et à radicaliser le régime directorial. Le but principal de ces clubs est "d'assurer le triomphe de la République en germinal" et un réseau de cercles, très dense dans le Centre-Ouest et le Sud-Ouest de la France, est constitué pendant l'hiver de l'an VI. Le cas de la Sarthe est bien documenté68. Un "cercle mobile" parcourt le département tous les décadis pour recruter des affiliés et, en ventôse (début de mars), une réunion générale a lieu au Mans pour préparer la campagne électorale. Dans l'Allier, on invite les inscrits au cercle à un banquet fraternel, où l'on distribue "la liste des personnes que l'on devait élire dans chacune des trois assemblées primaires de Moulins; chacun des membres a l'ordre de s'y conformer" ${ }^{69}$. Même chose en Charente : à Cognac, "on y établit un mode d'admission pour les candidats... on discute les qualités morales et politiques des individus qui pouvaient occuper les places... on propose un serment de n'élire que ceux qui ont obtenu le suffrage du cercle"70.

Les "royalistes" font la même chose et cette pratique trouve un reflet fidèle dans la presse du Directoire, surtout en l'an V. À Paris, La Quotidienne par exemple, essaie d'influencer les travaux de l'assemblée départementale qui s'ouvre dans la capitale le 20 germinal: “.. n'oubliez donc pas l'éloquence de Laharpe, Lacretelle, Richer, Quatremère de Quincy et Chauveau-Lagarde, des écrivains qui ont si longtemps lutté contre la tyrannie". À gauche, le Journal des hommes libres de tous les pays accuse ces mêmes individus d'incivisme. Cette guerre des plumes agite également les journaux de province. Dans le cas de Toulouse, par exemple, deux titres rivaux avancent leurs propres candidats et attaquent leurs adversaires, sous la rubrique: "Patriotes, ne perdez pas de vue germinal; occupez-vous des élections afin que vous fassiez de bons choix..." ${ }^{11}$.

C'est le bon vieux temps des candidatures officieuses, mais aux prises avec un phénomène nouveau: les candidats "officiels" du Directoire. À la suite du désastre électoral de l'an $\mathrm{V}$, les partisans du pouvoir décident de proposer leurs propres amis. Ils se servent de fonds secrets, distribués par des agents envoyés dans les départements (les soi-disant "commissaires des barrières), mais ils exploitent surtout le réseau administratif des commissaires du pouvoir exécutif qui se trouvent en place dans chaque canton. Le commissaire central de la Haute-Loire (nommé par le ministre de l'Intérieur et attaché à l'administration départementale), par exemple, devient effectivement l'agent électoral $\mathrm{du}$ gouvernement. Il encourage ses confrères à "travailler nuit et jour pour assurer "le triomphe de l'esprit républicain sur celui de Blankenbourg" (lieu de résidence du prétendant Louis XVIII) ${ }^{72}$. De nombreux commissaires (y compris une quarantaine en Haute-Loire) sont élus aux assemblées départementales de l'an VI, afin de soutenir les "candidats" directoriaux.

Il en va de même l'année suivante le commissaire central de la Corrèze est accusé d'avoir prétendu que, "quelques fussent les choix du peuple, le gouvernement n'accepterait que ceux qu'il avait fait désigner par un commissaire envoyé ad hoc dans 
le département"73. En même temps les autorités cherchent à réprimer les autres candidatures officieuses. Le journal de la Côte-d'Or décrit le déroulement de l'assemblée départementale de l'anVII dans la manière suivante :

"Ce n'est pas dans une grande assemblée uniquement occupée de mise ou de numération de votes qu'on peut présenter des candidats, les discuter, s'éclairer mutuellement et préparer de bons choix ; ainsi les électeurs, cette année comme les précédentes, s'étaient tous formés en réunions particulières pour composer une liste de sujets qui devait être examinée dans une assemblée générale."

En effet, sous la Révolution et après, on se représente le choix électoral comme une pratique collective, conduite en assemblée, à laquelle participe toute la communauté. On cherche à distinguer une volonté générale que la compétition électorale gâcherait en semant division et discorde. Le candidat imaginaire, qui transcende les intérêts particuliers et incarne des qualités idéales, reflète donc une répugnance envers les partis politiques, les programmes opposés et la concurrence personnelle comme éléments essentiels de l'offre et du choix électoraux. Durant le Directoire surtout, un autre discours fraye son chemin, anticipation du pluralisme, avant d'être étouffé par les graves crises politiques de la République libérale et foulé aux pieds par Bonaparte. Le processus d'acculturation électorale et de l'apprentissage de la culture politique pluraliste se réveillera au xixesiècle. Le refus obstiné des candidatures déclarées représente, au bout du compte, un aspect essentiel de la difficile naissance de la démocratie en France. 


\section{NOTES}

1.S. ABERDAM et al. (éds), Voter, élire pendant la Révolution française 1789-1799. Guide pour la recherche, Paris, 1999 P. GUENIFFEY, Le nombre et la raison. La Révolution française et les élections, Paris, 1993 М. СRоок, Elections in the French Revolution, 1789-1799: An Apprenticeship in Democracy, Cambridge, 1996.

2.F. A. AUlARD, Histoire politique de la Révolution française. Origines et développement de la démocratie et de la République (1789-1804), 4e éd. Paris, 1909, p.582.

3.Archives parlementaires, XV, p.704.

4.J. B. DUVERGIER (éd), Collection complète des lois, 24 vol., Paris, 1825-8, t.1, p.336.

5.G.SAUPIN, Nantes au XVIIe siècle. Vie politique et société urbaine, Rennes, 1996, par exemple.

6.M. CRоок, "The Persistence of the Ancien Régime in France : The Estates General of 1789 and the Origins of the Revolutionary Electoral System", Parliaments, Estates and Representation, 13, 1993, pp.29-40.

7.Déclaration interprétative de l'édit de mai 1765 pour l'administration des biens des villes. Je remercie Melvin Edelstein de cette référence.

8.Archives parlementaires, XXIX, p.367.

9.M.A. KISHLANSKY, Parliamentary Selection. Social and Political Choice in Early Modern England, Cambridge, 1986, pp. 9-21.

10.P. GUENIFFEY, op. cit., pp. 331-332.

11.Archives nationales (A.N.), C118, Procès-verbal d'élection, mai 1790.

12.Archives municipales (A.M.) de Toulon, L572, Procès-verbaux d'élection, février 1790.

13.T. MARGADANT, "Local Politics and Political Ambition during the French Revolution", dans M. ADCocK, E. CHESTER et J. WHITEMAN (éds), Revolution, Society and the Politics of Memory, Melbourne, 1998, p.131.

14.P. GUENIFFEY, op. cit., p. 331.

15.A.N., F1cIII Gironde 1, Adresse du directoire du département, 8 juin 1791.

16.Archives départementales (A.D.) de l'Aveyron, 1L582, Procès-verbal de Parizot, juin 1790.

17.A.N., BI 1, Procès-verbal d'élection, octobre 1790.

18.E. CharaVAy, Assemblée électorale de Paris, 3 vol., Paris, 1890-1905, t.2, pp. 528-529.

19.H. BAumont, "Les assemblées primaires et électorale de l'Oise en 1792 (août-

septembre), La Révolution française, XLVII (1904), pp. 164-171.

20.E. SEVESTRE, Les problèmes religieux de la Révolution et de l'Empire en Normandie, 1787-1815, Paris, 1924, p. 404.

21.A.N., F19 475, Assemblée départementale des Deux-Sèvres, 28 novembre 1790, 13 mars et 8 mai 1791.

22.P. BARREY, "Les élections à la Convention dans la Seine-Inférieure, La Révolution française, LXIV, 1913, p. 146.

23.A.D. Morbihan, L233, Procès-verbal d'élection, mai 1790.

24.P. GUENIFFEY, op. cit., p. 326.

25.A.D. Charente, L1681, Procès-verbal d'élection, octobre 1791.

26.J.-P. JESSENNE, "De la citoyenneté proclamée à la citoyenneté appliquée : l'exercice du droit de vote dans le District d'Arras en 1790, Revue du Nord, 72, 1990, p. 835. 
27.Bibliothèque nationale de France(B.N.F.), Lb39 10164, Projet d'instruction aux électeurs, 1791.

28.F. O'GORMAN, Voters, Patrons and Parties. The Unreformed Electorate of Hanoverian England, 1734-1832, Oxford, 1989, pp. 13-14.

29.P. GuenifFeY, op. cit., pp. 323 et sq.

30.E. CHARAVAY, op. cit., t.I, pp. XVI-XVII.

31.Id., t.II, p. 537.

32.C.-L. CHASSIN (éd), Les élections et les cahiers de doléances de Paris en 1789, 4 vol., Paris, 1888-1889, t.2, pp. 309-312.

33.Il est intéressant de remarquer que dans l'Oise, en 1792, deux candidats aux cures vacantes écrivent des "lettres de motivation, assorties de sorte de curriculum vitae. Elles sont reproduites par J.BERNET, “L'élection de l'évêque et des curés constitutionnels dans l'Oise sous la Révolution (1791-1793), Annales historiques compiégnoises, 71-72, 1998, pp. 34-35.

34.A. RochAs, Journal d'un bourgeois de Valence, 2 vol., Grenoble, 1891, t. 2, p. 185.

35.B.N.F., Lb39 10166, Petit traité de la cabale, ou l'art d'accaparer les suffrages, à l'usage des MM. les électeurs, 1791.

36.A.M. Toulon, L579, Procès-verbaux d'élection, novembre 1791.

37.M. KenNEDY, The Jacobin Clubs in the French Revolution, 2 vol., Princeton, 1982-1988, t.1, pp.210 et $s q$.

38.A.D. Bouches-du-Rhône, L1964, Pétition de J. Autran, 11 janvier 1793.

39.Voir E. CHARAVAY, op. cit., t.III, pp. 591-614 et M. CROOK, "La plume et l'urne : la presse et les élections sous le Directoire, dans P. Bourdin et B. GAINOT (éds), La République directoriale, 2 vol., Paris, 1998, t.1, pp. 296-300.

40.P. GUENIFFEY, op. cit., pp. 343 et $s q$.

41.Journal des départements méridionaux, t.2, p. 353, 15 septembre 1792.

42.H. BAUMONT, op. cit., p. 154.

43.P. GUENIFFEY, op. cit., p. 315.

44.Archives parlementaires, LXIII, pp. 309-310.

45.J. V. DELACROIX, Le Spectateur françois pendant le gouvernement révolutionnaire, Paris, an III, pp. 232-237.

46.F. A. AUlaRd (éd), Recueil de documents pour l'histoire du Club des Jacobins, 6 vol., Paris, 1889-1897, t. 2, p. 389.

47.A.N., DIV 28, Société des Amis de la Constitution de Quimper, 11 mai 1791.

48.A.N., F1cIII Lot-et-Garonne 1, Arrêté de l'administration centrale, 6 frimaire an V (26 novembre 1796).

49.Moniteur, XXV, p. 710.

50.A.D. Côte-d'Or, L212, Liste des candidats inscrits, 23 pluviôse an V (11 février 1797) A.N., F1cIII Gironde 1 (et aussi A.M. Bordeaux, K4), Tableau des citoyens, 15 ventôse an V (5 mars 1797) A.D. Lot-et-Garonne, L211, Liste des candidats inscrits, 15 pluviôse an V (3 février 1797) A.D. Loire-Atlantique, L173, Liste des citoyens désignés par les cantons, 23 pluviôse an V (11 février 1797) A.D. Meurthe, L91, Registre des délibérations de l'administration du département, 25 pluviôse an V (13 février 1797) A.D. Yvelines, 1LM370, Liste alphabétique des candidats, 7 ventôse an V (25 février 1797) et A.FRAYFOURNIER (éd), Le département de la Haute-Vienne. Archives modernes, 2e série, t. 7 (Limoges, 1909), pp.150-153. On retrouve aux A.N. une liste pour le Bas-Rhin, mais elle se restreint 
aux postes législatifs (A.N. F1cIII Bas-Rhin 1, Liste des candidats, 21 pluviôse an V - 9 février 1797).

51.B.N.F., Le 40 129, Tableau des citoyens de la commune de Nantes indiqués pour candidats, 4nivôse V (24 déc. 1796) A.D. Cantal, L143, Listes des candidats, pluviôse et ventôse an V (janvier - mars 1797) A.D. Yvelines, 1LM370, Procès-verbaux d'élection, germinal an V (mars 1797) et R. MARX, Recherches sur la vie politique de l'Alsace prérévolutionnaire et révolutionnaire, Strasbourg, 1966, p. 164.

52.A.D. Cantal, L143, Listes des candidats, et A.D. Côtes-d'Armor, 1L411, Listes des candidats, nivôse et pluviôse an V (décembre 1796 - février 1797).

53.A.D. Yvelines, 1LM370, Procès-verbaux d'élection, germinal an V (mars 1797), et P.GUENIFFEY, op. cit., p. 504.

54.A.D. Finistère, $10 \mathrm{~L} 85$, Administration cantonale, pluviôse an V (janvier - février 1797).

55.A.D. Alpes de Haute-Provence, L202, Procès-verbal d'élection, 1 germinal an V (21 mars 1797). Idem dans la Meurthe selon P. CLÉMENDOT, Le département de la Meurthe à l'époque du Directoire, Nancy, 1966, pp. 223-224.

56.A.N., F1cIII Lot-et-Garonne 1, Arrêté de l'administration centrale.

57.Y. LE GALL, "Les consultations générales en Loire-Inférieure, 1789-an VII, Thèse pour le Doctorat d'État en Droit, Université de Nantes, 2 vol., 1976, t.2, p. 743.

58.Pour Paris on trouve la mention dans A.N., AF III 261, Commissaire central au ministre, 27germinal an V (16 avril 1797).

59.Y. LE GALL, op. cit., t.2, p. 744.

60.Id., t.2, pp. 745-749.

61.M. REINHARD, Le département de la Sarthe sous le régime directorial, Saint-Brieuc, 1935, pp. 257-258. Idem dans les Côtes-du-Nord : H. POMMERET, L'esprit public dans le département des Côtes-du-Nord pendant la Révolution, 1789-1799, Saint-Brieuc, 1921, pp. 384-385.

62.PONS DE VERDUN, Rapport... sur la suppression des listes de candidats, Paris, an VI.

63.LEBRETON, Rapport au Conseil des Anciens, Paris, an VI.

64.A.C. QUATREMÈRE DE QUINCY, La véritable liste des candidats, Paris, an V.

65.PONS DE VERDUN, Rapport sur la suppression du scrutin de rejet, Paris, an VI.

66.FRAY-FOURNIER, op. cit., p. 157 et Patrice GUENIFFEY, op. cit., p. 492.

67.M. CROOK, Elections (...), op. cit., pp. 147-157.

68.M. REINHARD, op. cit., p.319.

69.A.N., AF III 212, Commissaire du directoire, 7 germinal VI (27 mars 1798).

70.A.N., AF III 219, Rapport du directoire du département, sans date.

71.M. CROOK, "La plume (...), op. cit., pp. 300-310.

72.E. DelCAMBRE, La période du Directoire en Haute-Loire, 3 vol., Rodez, 1941-1943, t. 2, p. 254.

73.A.N., F1cIII Corrèze 4, Au Directoire exécutif, 7 germinal VII (27 mars 1799).

74.A.N., F1cIII Côte-d'Or 2, Extrait du Journal de la Côte-d'Or, 30 germinal VII (19 avril 1799).

75.S. KENT, The Election of 1827 in France, Cambridge, Mass., 1975, pp. 122-124.

76.A. LEFÈVRE-PONTALIS, Les lois et les mours électorales en France et en Angleterre, Paris, 1864, p. 18.

77.Loi du 17 juillet 1889 relative aux candidatures multiples. 


\section{RÉSUMÉS}

Les candidats dans les élections de la période révolutionnaire sont "imaginaires" dans le sens où ils existent dans les principes plus que dans la pratique électorale. Hormis lors de l'expérience unique de l'an V (avec des candidats déclarés) qui est examinée ici, on se méfie des candidatures publiques aux élections de la décennie révolutionnaire. Selon les contemporains, la vraie candidature n'a besoin ni de programme, ni de publicité, elle réside plutôt dans le caractère et la réputation. Un choix électoral illimité, pourtant, pose de graves problèmes: dispersion des voix, refus d'accepter le mandat et prolongation du processus électoral. Mais si l'idée des candidatures publiques est vite abandonnée, sous le Directoire on assiste à la naissance des candidats officieux.Cette pratique des campagnes électorales choque les esprits qui resteront longtemps attachés au mythe de la volonté générale indivisible.

\section{Imaginary candidates or candidatures and choices in the elections of the French Revolution}

Candidates in the elections of the French Revolution were "imaginary " in the sense that they had greater reality in theory than in practice. Apart from the isolated experiment with declared candidatures in the Year V, which is examined in this article, there was great reluctance to accept the idea of candidates during the revolutionary decade. The real candidature was not to be found in programmes and publicity materials, but in character and reputation. An unlimited choice for voters, however, produced severe problems: the dispereal of votes, refusals to take office and a prolongation of the electoral process. Yet, though public candidatures were quickly abandoned after 1797, for the remainder of the Directory « unofficial » candidates were much in evidence. The emergence of canvassing and campaigning shocked contemporaries, who remained deeply attached to the myth of the undivided general will.

\section{INDEX}

Mots-clés : révolution, élections, candidats

\section{AUTEUR}

\section{MALCOLM CROOK}

Université de Keele 